\title{
Controllability for systems governed by semilinear evolution inclusions without compactness
}

\author{
Irene Benedetti, Valeri Obukhovskii and Valentina Taddei
}

\begin{abstract}
In this paper we study the controllability for a class of semilinear differential inclusions in Banach spaces. Since we assume the regularity of the nonlinear part with respect to the weak topology, we do not require the compactness of the evolution operator generated by the linear part. As well we are not posing any conditions on the multivalued nonlinearity expressed in terms of measures of noncompactness. We are considering the usual assumption on the controllability of the associated linear problem. Notice that, in infinite dimensional spaces, the above mentioned compactness of the evolution operator and linear controllability condition are in contradiction with each other. We suppose that the nonlinear term has convex, closed, bounded values and a weakly sequentially closed graph when restricted to its second argument. This regularity setting allows us to solve controllability problem under various growth conditions. As application, a controllability result for hyperbolic integro-differential equations and inclusions is obtained. In particular, we consider controllability of a system arising in a model of nonlocal spatial population dispersal and a system governed by the second order one-dimensional telegraph equation.
\end{abstract}

Mathematics Subject Classification (2010). Primary 34G25, 93B05;

Secondary 34B15, 47H04, 28B20.

Keywords. Semilinear evolution equations and inclusions, Controllability, Fixed point theorems.

\section{Introduction}

This paper deals with the controllability for a class of semilinear differential inclusions in a reflexive Banach space. Precisely, let $[a, b]$ be a fixed interval of the real line, we investigate the following control system:

$$
\left\{\begin{array}{l}
x^{\prime}(t) \in A(t) x(t)+F(t, x(t))+B u(t) \text { a.e. } t \in[a, b], \\
x(a)=x_{0},
\end{array}\right.
$$


where $\{A(t)\}_{t \in[a, b]}$ is a family of linear not necessarily bounded operators, generating an evolution operator; $F:[a, b] \times E \multimap E$ is a multivalued map, the control function $u(\cdot)$ is considered in the space $L^{2}([0, b] ; U)$, where $U$ is a Banach space of controls and $B: U \rightarrow E$ is a bounded linear operator.

We will consider the controllability problem for this system, i.e., we will study conditions under which there exists a trajectory $x(\cdot)$ of the above system reaching a given state at the final time $b$.

The above problem is largely studied in literature. See, e.g. $[3,4,12,14,19$, $25]$ and the references therein. It is worth noting also that differential inclusions represent a useful and convenient tool for describing various optimal control problems (see, e.g. $[1,2,20,22]$ ).

A usual assumption to obtain controllability for nonlinear problems is the controllability for the associated linear ones. As it was pointed out by Triggiani in [32] and [33], in infinite dimensional Banach spaces the compactness of the associated evolution operator is in contradiction with the controllability of a linear system while using locally $L^{p}$ - controls, for $p>1$. On the other hand, a typical application of abstract differential equations and inclusions is to consider them as the abstract form of a partial differential equation. In this framework sufficient conditions for the controllability of the associated linear problem are usually given for controls in $L^{2}$. Thus, it is meaningful to introduce conditions assuring controllability for semilinear equations without requiring the compactness of the semigroup or evolution operator generated by the linear part.

A first contribution in this direction is related with a regularity assumption on the non-linear term, formulated through a measure of non-compactness, see [27], and [8] for the case of impulsive semilinear differential inclusions.

In this paper another approach is considered, it exploits the weak topology of the state space. This new tool was introduced to study semilinear differential inclusions associated to various boundary value conditions, see $[5,6]$ and [7]. In this paper we show that the same approach can be applied to obtain the controllability in finite time for such problems. We stress that this technique allows to consider both sublinear and superlinear growth condition on the nonlinear term.

In the last section some applications arising from physics and biology are provided. More precisely, we apply the main theorem of the paper (Theorem 4.1) to obtain the controllability in two models. The first one is a model in nonlocal spatial dispersal, arising in biology and in the theory of phase transition as a generalization of the classical diffusion. Indeed the introduction of a dispersal kernel well describes the model, because it takes into account the long-distance dispersal (see $[11,13]$ ). The second one is a system governed by a model of a one-dimensional telegraph equation with constant coefficients. The telegraph equation is important for modeling several relevant problems such as the vibrations of structures (e.g., buildings, beams, and machines) and it is commonly used in signal analysis for transmission and propagation of electrical signals. Recently, telegraph equation became more suitable than ordinary dif- 
fusion equation in modeling the reaction diffusion for such branches of sciences $($ see $[26,30])$.

The paper is organized as follows. In Sect. 2 we recall some notions and results that we use in the main part of the paper. In Sect. 3 we state the problem with the main assumptions, in Sect. 4 we prove the controllability for the abstract problem and in Sect. 5 we demonstrate the controllability for the class of partial differential equations mentioned above.

\section{Preliminaries}

Let $(E,\|\cdot\|)$ be a reflexive Banach space and $E_{w}$ denote the space $E$ endowed with the weak topology. We denote by $n B$ the closed ball of $E$ centered at the origin and of radius $n$ and, for a set $A \subset E$, the symbol $\bar{A}^{w}$ denotes the weak closure of $A$. We recall that a bounded subset $A$ of a reflexive Banach space $E$ is weakly relatively compact. In the whole paper, without generating misunderstanding, we denote by $\|\cdot\|_{p}$ both the $L^{p}([a, b] ; E)$-norm and $L^{p}([a, b] ; \mathbb{R})$-norm and by $\|\cdot\|_{0}$ the $C([a, b] ; E)$-norm. We recall (see $[9$, Theorem 4.3$]$ ) that a sequence $\left\{x_{n}\right\} \subset C([a, b] ; E)$ weakly converges to an element $x \in C([a, b] ; E)$ if and only if

1. There exists $N>0$ such that, for every $n \in \mathbb{N}$ and $t \in[a, b],\left\|x_{n}(t)\right\| \leq N$;

2. For every $t \in[a, b], x_{n}(t) \rightarrow x(t)$.

Put $\Delta=\{(t, s) \in[a, b] \times[a, b]: a \leq s \leq t \leq b\}$, we recall (see, e.g. [23, $28])$ that a two parameter family of bounded linear operators $\{T(t, s)\}_{(t, s) \in \Delta \text {, }}$ $T(t, s): E \rightarrow E$, is called an evolution system if the following conditions are satisfied:

1. $T(s, s)=I, a \leq s \leq b ; \quad T(t, r) T(r, s)=T(t, s), a \leq s \leq r \leq t \leq b ;$

2. For each $x \in E$, the function $(t, s) \in \Delta \rightarrow T(t, s) x$ is continuous.

To every evolution system we can assign the corresponding evolution operator $T: \Delta \rightarrow \mathcal{L}(E)$, where $\mathcal{L}(E)$ is the space of all bounded linear operators in $E$.

It is known (see, e.g., [23]) that there exists a constant $M=M_{\Delta}>0$ such that

$$
\|T(t, s)\|_{\mathcal{L}(E)} \leq M, \quad \forall(t, s) \in \Delta .
$$

Finally, for sake of completeness, we recall some results that we will need in the main section.

Firstly we state the Glicksberg-Ky Fan fixed point Theorem [18,24].

Theorem 2.1. Let $X$ be a Hausdorff locally convex topological vector space, $K$ a compact convex subset of $X$ and $G: K \multimap K$ an upper semicontinuous multimap with closed, convex values. Then $G$ has a fixed point $x_{*} \in K: x_{*} \in$ $G\left(x_{*}\right)$.

We mention also two results that are contained in the so called EberleinSmulian theory. 
Theorem 2.2. [21, Theorem 1, p. 219] Let $\Omega$ be a subset of a Banach space $X$. The following statements are equivalent:

1. $\Omega$ is relatively weakly compact;

2. $\Omega$ is relatively weakly sequentially compact.

Corollary 2.1. [21, p. 219] Let $\Omega$ be a subset of a Banach space X. The following statements are equivalent:

1. $\Omega$ is weakly compact;

2. $\Omega$ is weakly sequentially compact.

We recall the Krein-Smulian Theorem.

Theorem 2.3. [15, p. 434] The convex hull of a weakly compact set in a Banach space $E$ is weakly compact.

In conclusion we recall the Pettis measurability Theorem which we use in Sect. 5 .

Theorem 2.4. [29, p. 278] Let $(X, \Sigma)$ be a measure space, $E$ be a separable Banach space. Then a function $f: X \rightarrow E$ is measurable if and only if for every $e \in E^{\prime}$ the function $e \circ f: X \rightarrow \mathbb{R}$ is measurable with respect to $\Sigma$ and the Borel $\sigma$-algebra in $\mathbb{R}$.

\section{Problem statement}

We study the controllability problem for a system governed by inclusion (1.1) under the following assumptions.

(A) $\{A(t)\}_{t \in[a, b]}$ is a family of linear not necessarily bounded operators, generating an evolution operator, i.e. $A(t): D(A) \subset E \rightarrow E$, with $D(A)$ a dense subset of $E$ not depending on $t \in[a, b]$ and there exists an evolution system $\{T(t, s)\}_{(t, s) \in \Delta}$ with $T: \Delta \rightarrow \mathcal{L}(E)$ strongly differentiable (see, e.g. [23]) with respect to $t$ and $s$, precisely

$\frac{\partial T(t, s)}{\partial t}=A(t) T(t, s) \quad$ and $\quad \frac{\partial T(t, s)}{\partial s}=-T(t, s) A(s), \quad(t, s) \in \Delta$.

We assume that the multivalued nonlinearity $F:[a, b] \times E \multimap E$ has closed bounded and convex values and:

(F1) The multifunction $F(\cdot, c):[a, b] \multimap E$ has a measurable selection for every $c \in E$, i.e., there exists a measurable function $f:[a, b] \rightarrow E$ such that $f(t) \in F(t, c)$ for a.e. $t \in[a, b]$;

(F2) The multimap $F(t, \cdot): E \multimap E$ is weakly sequentially closed for a.e. $t \in[a, b]$, i.e. it has a weakly sequentially closed graph;

(F3) For every $r>0$ there exists a function $\mu_{r} \in L^{1}\left([a, b] ; \mathbb{R}_{+}\right)$such that for each $c \in E,\|c\| \leq r$ :

$$
\|F(t, c)\|=\sup \{\|x\|: x \in F(t, c)\} \leq \mu_{r}(t) \text { for a.e. } t \in[a, b] ;
$$


(B) The control function $u(\cdot)$ belongs to the space $L^{2}([a, b] ; U)$, where $U$ is a Banach space of controls, and $B: U \rightarrow E$ is a bounded linear operator, with

$$
\|B\|=M_{1} .
$$

Definition 3.1. A continuous function $x:[a, b] \rightarrow E$ is a mild solution to problem (1.1) if $x$ may be represented in the following form:

$$
x(t)=T(t, a) x_{0}+\int_{a}^{t} T(t, s) f(s) d s+\int_{a}^{t} T(t, s) B u(s) d s, \quad t \in[a, b]
$$

where $f \in L^{1}([a, b] ; E), f(s) \in F(s, x(s))$ for a.a. $s \in[a, b]$, and $u \in L^{2}([a, b] ; U)$.

We will consider the controllability problem for the above system, i.e., we will study conditions which guarantee the existence of a mild solution to problem (1.1) satisfying

$$
x(b)=x_{1}
$$

where $x_{1} \in E$ is a given point. A pair $(x, u)$ consisting of a mild solution $x(\cdot)$ to (1.1) satisfying (3.2) and of the corresponding control $u(\cdot) \in L^{2}([a, b] ; E)$ is called a solution of the controllability problem.

We assume the standard assumption that the corresponding linear problem (i.e. when $F(t, c) \equiv 0$ ) has a solution. More precisely, we suppose that $(\mathrm{W})$ the controllability operator $W: L^{2}([a, b] ; U) \rightarrow E$ given by

$$
W u=\int_{a}^{b} T(b, s) B u(s) d s
$$

has a bounded inverse $W^{-1}: E \rightarrow L^{2}([a, b] ; U) / \operatorname{Ker}(W)$.

It should be mentioned that we may assume, w.l.o.g., that $W^{-1}$ acts into $L^{2}([a, b] ; U)$ (see, e.g., [3], p. 9). Denote

$$
\left\|W^{-1}\right\|=M_{2}
$$

\section{Controllability}

Given $q \in C([a, b] ; E)$, let us denote

$$
\mathfrak{S}_{q}=\left\{f \in L^{1}([a, b] ; E): f(t) \in F(t, q(t)) \text { for a.a. } t \in[a, b]\right\} .
$$

The set $\mathfrak{S}_{q}$ is always nonempty as Proposition 4.1 below shows. In the proof we will need the following auxiliary assertion.

Lemma 4.1. ([6, Proposition 2.2]) Let $F:[a, b] \times E \multimap E$ be a multimap satisfying properties (F1) and (F3). If $F(t, \cdot): E \multimap E_{w}$ is upper semicontinuous for a.e. $t \in[a, b]$, then the set $\mathfrak{S}_{q}$ is nonempty for any $q \in C([a, b] ; E)$.

Proposition 4.1. For a multimap $F:[a, b] \times E \multimap E$ satisfying properties (F1), (F2) and (F3), the set $\mathfrak{S}_{q}$ is nonempty for any $q \in C([a, b] ; E)$. 
Proof. By (F3) the multimap $F(t, \cdot)$ is locally weakly compact for a.e. $t \in[a, b]$, i.e. for a.e. $t$ and every $x \in E$ there is a neighbourhood $V$ of $x$ such that the restriction of $F(t, \cdot)$ to $V$ is weakly compact. Hence by (F2) and the locally weak compactness, we easily get that $F(t, \cdot): E_{w} \multimap E_{w}$ is upper semicontinuous for a.e. $t \in[a, b]$. Thus, $F(t, \cdot): E \multimap E_{w}$ is upper semicontinuous for a.e. $t \in[a, b]$ and the thesis follows by Lemma 4.1.

We denote with $S_{1}: L^{1}([a, b] ; E) \rightarrow C([a, b] ; E)$ and $S_{2}: L^{1}([a, b] ; E) \rightarrow$ $C([a, b] ; E)$ the following integral operators

$$
\begin{aligned}
& S_{1} f(t)=\int_{a}^{t} T(t, s) f(s) d s \quad \forall t \in[a, b] \\
& S_{2} f(t)=\int_{a}^{t} T(t, s) B W^{-1}\left(-\int_{a}^{b} T(b, \eta) f(\eta) d \eta\right)(s) d s \quad \forall t \in[a, b] .
\end{aligned}
$$

We define the solution multioperator $\Gamma: C([a, b] ; E) \multimap C([a, b] ; E)$ as

$$
\Gamma(q)=\left\{\begin{array}{l}
x \in C([a, b] ; E): \\
x(t)=T(t, a) x_{0}+S_{1} f(t)+\int_{a}^{t} T(t, s) \\
B W^{-1}\left(x_{1}-T(b, a) x_{0}\right)(s) d s+S_{2} f(t), f \in \mathfrak{S}_{q}
\end{array}\right\} .
$$

It is easy to verify that the fixed points of the multioperator $\Gamma$ are mild solutions of controllability problem (1.1), (3.2).

Lemma 4.2. The operators $S_{1}$ and $S_{2}$ defined in (4.1) are linear and continuous.

Proof. The linearity follows from the linearity of the integral operator and of the operators $B, W^{-1}$ and $T(t, s)$ for every $(t, s) \in \Delta$. By (2.1) we have

$$
\left\|S_{1} f(t)\right\|=\left\|\int_{a}^{t} T(t, s) f(s) d s\right\| \leq M\|f\|_{1}, \quad \forall t \in[a, b],
$$

and moreover by (3.1), (3.3) we obtain

$$
\begin{aligned}
\left\|S_{2} f(t)\right\| & \leq\left\|\int_{a}^{t} T(t, s) B W^{-1}\left(-\int_{a}^{b} T(b, \eta) f(\eta) d \eta\right)(s) d s\right\| \\
& \leq M M_{1} \int_{a}^{t}\left\|W^{-1}\left(-\int_{a}^{b} T(b, \eta) f(\eta) d \eta\right)(s)\right\| d s \\
& \leq M M_{1}\left\|W^{-1}\left(-\int_{a}^{b} T(b, \eta) f(\eta) d \eta\right)\right\|_{L^{1}([a, b] ; U)} \\
& \leq M M_{1} \sqrt{b-a}\left\|W^{-1}\left(-\int_{a}^{b} T(b, \eta) f(\eta) d \eta\right)\right\|_{L^{2}([a, b] ; U)} \\
& \leq M M_{1} M_{2} \sqrt{b-a}\left(M \int_{a}^{b}\|f(\eta)\| d \eta\right) \\
& =M^{2} M_{1} M_{2} \sqrt{b-a}\|f\|_{1}, \quad \forall t \in[a, b] .
\end{aligned}
$$


Fix $n \in \mathbb{N}$, consider $Q_{n}$ the closed ball of radius $n$ in $C([a, b] ; E)$ centered at the origin and denote by $\Gamma_{n}=\Gamma\left\lfloor_{Q_{n}}: Q_{n} \multimap C([a, b] ; E)\right.$ the restriction of the multioperator $\Gamma$ on the set $Q_{n}$. We describe some properties of $\Gamma_{n}$.

Proposition 4.2. The multioperator $\Gamma_{n}$ has a weakly sequentially closed graph.

Proof. Let $\left\{q_{m}\right\} \subset Q_{n}$ and $\left\{x_{m}\right\} \subset C([a, b] ; E)$ satisfying $x_{m} \in \Gamma_{n}\left(q_{m}\right)$ for all $m$ and $q_{m} \rightarrow q, x_{m} \rightarrow x$ in $C([a, b] ; E)$; we will prove that $x \in \Gamma_{n}(q)$.

Since $q_{m} \in Q_{n}$ for all $m$ and $q_{m}(t) \rightarrow q(t)$ for every $t \in[a, b]$, it follows that $\|q(t)\| \leq \liminf _{m \rightarrow \infty}\left\|q_{m}(t)\right\| \leq n$ for all $t$ (see [10, Proposition III.5]). The fact that $x_{m} \in \Gamma_{n}\left(q_{m}\right)$ means that there exists a sequence $\left\{f_{m}\right\}, f_{m} \in \mathfrak{S}_{q_{m}}$, such that for every $t \in[a, b]$,

$x_{m}(t)=T(t, a) x_{0}+S_{1} f_{m}(t)+\int_{a}^{t} T(t, s) B W^{-1}\left(x_{1}-T(b, a) x_{0}\right)(s) d s+S_{2} f_{m}(t)$.

We observe that, according to (F3), $\left\|f_{m}(t)\right\| \leq \eta_{n}(t)$ for a.a. $t$ and every $m$, i.e. $\left\{f_{m}\right\}$ is bounded and uniformly integrable and $\left\{f_{m}(t)\right\}$ is bounded in $E$ for a.a. $t \in[a, b]$. Hence, by the reflexivity of the space $E$ and by the DunfordPettis Theorem (see [15, p. 294]), we have the existence of a subsequence, denoted as the sequence, and a function $g$ such that $f_{m} \rightarrow g$ in $L^{1}([a, b] ; E)$.

Therefore, we have that $S_{i} f_{m} \rightarrow S_{i} g$ for $i=1,2$. Thus

$$
\begin{aligned}
& x_{m}(t) \rightarrow T(t, a) x_{0}+S_{1} g(t)+\int_{a}^{t} T(t, s) B W^{-1} \\
& \quad \times\left(x_{1}-T(b, a) x_{0}\right)(s) d s+S_{2} g(t)=x_{0}(t), \quad \forall t \in[a, b]
\end{aligned}
$$

implying, for the uniqueness of the weak limit in $E$, that $x_{0}(t)=x(t)$ for all $t \in[a, b]$.

To conclude, we have only to prove that $g(t) \in F(t, q(t))$ for a.a. $t \in[a, b]$.

By Mazur's convexity Theorem (see e.g. [16]) we have a sequence

$$
\tilde{f}_{m}=\sum_{i=0}^{k_{m}} \lambda_{m i} f_{m+i}, \quad \lambda_{m i} \geq 0, \sum_{i=0}^{k_{m}} \lambda_{m i}=1
$$

satisfying $\tilde{f}_{m} \rightarrow g$ in $L^{1}([a, b] ; E)$ and, up to subsequence, there is $N_{0} \subset[a, b]$ with Lebesgue measure zero such that $\tilde{f}_{m}(t) \rightarrow g(t)$ for all $t \in[a, b] \backslash N_{0}$ (see [31, Chapter IV, Theorem 38]). With no loss of generality we can also assume that $F(t, \cdot): E_{w} \multimap E_{w}$ is weakly sequentially closed and $\sup _{\|F(t, x)\| \leq}$ $\eta_{n}(t)$ for every $t \notin N_{0}$.

Fix $t_{0} \notin N_{0}$ and assume, by contradiction, that $g\left(t_{0}\right) \notin F\left(t_{0}, q\left(t_{0}\right)\right)$. By the reflexivity of the space $E$ and (F3) the restriction $F_{n B}\left(t_{0}, \cdot\right)$ of the multimap $F\left(t_{0}, \cdot\right)$ on the set $n B$ is weakly compact. Hence, by Corollary 2.1, we have that $F_{n B}\left(t_{0}, \cdot\right)$ is a weakly closed multimap and by $[20$, Theorem 
1.1.5] it is weakly u.s.c. Since $\left\|q\left(t_{0}\right)\right\| \leq n$ and since $F_{n B}\left(t_{0}, q\left(t_{0}\right)\right)$ is closed and convex, from the Hahn-Banach Theorem there is a weakly open convex set $V \supset F_{n B}\left(t_{0}, q\left(t_{0}\right)\right)$ satisfying $g\left(t_{0}\right) \notin \bar{V}^{w}$. Being $F_{n B}\left(t_{0}, \cdot\right)$ weakly u.s.c., we can also find a weak neighbourhood $V_{1}$ of $q\left(t_{0}\right)$ such that $F_{n B}\left(t_{0}, x\right) \subset V$ for all $x \in V_{1}$ with $\|x\| \leq n$. Notice that $\left\|q_{m}\left(t_{0}\right)\right\| \leq n$ for all $m$. The convergence $q_{m}\left(t_{0}\right) \rightarrow q\left(t_{0}\right)$ as $m \rightarrow \infty$ then implies the existence of $m_{0} \in \mathbb{N}$ such that $q_{m}\left(t_{0}\right) \in V_{1}$ for all $m>m_{0}$. Therefore $f_{m}\left(t_{0}\right) \in F_{n B}\left(t_{0}, q_{m}\left(t_{0}\right)\right) \subset V$ for all $m>m_{0}$. The convexity of $V$ implies that $\tilde{f}_{m}\left(t_{0}\right) \in V$ for all $m>m_{0}$ and, by the convergence, we arrive to the contradictory conclusion that $g\left(t_{0}\right) \in \bar{V}^{w}$. We obtain that $g(t) \in F(t, q(t))$ for a.a. $t \in[a, b]$.

Proposition 4.3. The multioperator $\Gamma_{n}$ is weakly compact.

Proof. We first prove that $\Gamma_{n}\left(Q_{n}\right)$ is weakly relatively sequentially compact.

Let $\left\{q_{m}\right\} \subset Q_{n}$ and $\left\{x_{m}\right\} \subset C([a, b] ; E)$ satisfying $x_{m} \in \Gamma_{n}\left(q_{m}\right)$ for all $m$. By the definition of the multioperator $\Gamma_{n}$, there exist a sequence $\left\{f_{m}\right\}$, $f_{m} \in \mathfrak{S}_{q_{m}}$, such that

$$
\begin{aligned}
x_{m}(t)= & T(t, a) x_{0}+S_{1} f_{m}(t)+\int_{a}^{t} T(t, s) B W^{-1} \\
& \times\left(x_{1}-T(b, a) x_{0}\right)(s) d s+S_{2} f_{m}(t), \quad \forall t \in[a, b] .
\end{aligned}
$$

Further, reasoning as in Proposition 4.2, we have that there exists a subsequence, denoted as the sequence, and a function $g$ such that $f_{m} \rightarrow g$ in $L^{1}([a, b] ; E)$. Therefore

$$
\begin{aligned}
x_{m}(t) \rightarrow l(t)= & T(t, a) x_{0}+S_{1} g(t)+\int_{a}^{t} T(t, s) B W^{-1}\left(x_{1}-T(b, a) x_{0}\right)(s) d s \\
& +S_{2} g(t), \quad \forall t \in[a, b] .
\end{aligned}
$$

Furthermore, by the weak convergence of $\left\{f_{m}\right\}$, by (2.1), (3.1), (3.3), and the continuity of the operators $S_{1}$ and $S_{2}$ we have

$$
\begin{aligned}
\left\|x_{m}(t)\right\| \leq & M\left\|x_{0}\right\|+M\left\|\eta_{n}\right\|_{1}+M M_{1} M_{2} \sqrt{b-a}\left(\left\|x_{1}\right\|+M\left\|x_{0}\right\|\right) \\
& +M^{2} M_{1} M_{2} \sqrt{b-a}\left\|\eta_{n}\right\|_{1}
\end{aligned}
$$

for all $m \in \mathbb{N}$ and for all $t \in[a, b]$. Reasoning again like in Proposition 4.2, it is then easy to prove that $x_{m} \rightarrow l$ in $C([a, b] ; E)$. Thus $\Gamma_{n}\left(Q_{n}\right)$ is weakly relatively sequentially compact, hence weakly relatively compact by Theorem 2.2 .

Proposition 4.4. The multioperator $\Gamma_{n}$ has convex and weakly compact values.

Proof. Fix $q \in Q_{n}$, since $F$ is convex valued, from the linearity of the integral and of the operators $T(t, s)$ for all $(t, s) \in \Delta, B$ and $W^{-1}$ it follows that the set $\Gamma_{n}(q)$ is convex. The weak compactness of $\Gamma_{n}(q)$ follows by Propositions 4.2 and 4.3 .

We are able now to state the main result of this section.

Theorem 4.1. Under assumptions $(A),(F 1),(F 2),(B),(W)$ and 
$\left(F 3^{\prime}\right)$ there exists a sequence of functions $\left\{\omega_{n}\right\} \subset L^{1}\left([a, b] ; \mathbb{R}_{+}\right)$such that

$$
\sup _{\|c\| \leq n}\|F(t, c)\| \leq \omega_{n}(t) \text { for a.a. } t \in[a, b], n \in \mathbb{N}
$$

with

$$
\liminf _{n \rightarrow \infty} \frac{1}{n} \int_{a}^{b} \omega_{n}(s) d s=0,
$$

controllability problem (1.1), (3.2) has a solution.

Proof. We show that there exists $n \in \mathbb{N}$ such that the operator $\Gamma_{n}$ maps the ball $Q_{n}$ into itself.

Assume to the contrary, that there exist sequences $\left\{z_{n}\right\},\left\{y_{n}\right\}$ such that $z_{n} \in Q_{n}, y_{n} \in \Gamma_{n}\left(z_{n}\right)$ and $y_{n} \notin Q_{n}, \forall n \in \mathbb{N}$. Then there exists a sequence $\left\{f_{n}\right\} \subset L^{1}([a, b] ; E), f_{n}(s) \in F\left(s, z_{n}(s)\right) \forall n \in \mathbb{N}$ and a.a. $s \in[a, b]$ such that

$$
\begin{aligned}
y_{n}(t)= & T(t, a) x_{0}+S_{1} f_{n}(t)+\int_{a}^{t} T(t, s) \\
& \times B W^{-1}\left(x_{1}-T(b, a) x_{0}\right)(s) d s+S_{2} f_{n}(t), \forall t \in[a, b] .
\end{aligned}
$$

Reasoning as in Proposition 4.3 we have

$$
\left\|y_{n}\right\|_{0} \leq C_{1}+C_{2}\left(\int_{a}^{b}\left\|f_{n}(\eta)\right\| d \eta\right) \leq C_{1}+C_{2}\left(\int_{a}^{b} \omega_{n}(\eta) d \eta\right),
$$

where

$$
\begin{gathered}
C_{1}=M\left\|x_{0}\right\|+M M_{1} M_{2} \sqrt{b-a}\left(\left\|x_{1}\right\|+M\left\|x_{0}\right\|\right) \\
C_{2}=M\left(1+M M_{1} M_{2} \sqrt{b-a}\right) .
\end{gathered}
$$

But then

$$
1<\frac{\left\|y_{n}\right\|_{0}}{n} \leq \frac{C_{1}}{n}+\frac{C_{2}}{n} \int_{a}^{b} \omega_{n}(\eta) d \eta, \quad n \in \mathbb{N}
$$

giving the contradiction with (4.3).

Fix, now, $n \in \mathbb{N}$ such that $\Gamma_{n}\left(Q_{n}\right) \subseteq Q_{n}$. By Proposition 4.3 the set $V_{n}=$ $\overline{\Gamma_{n}\left(Q_{n}\right)}{ }^{w}$ is weakly compact. Let now $W_{n}=\overline{\mathrm{co}}\left(V_{n}\right)$, where $\overline{\mathrm{co}}\left(V_{n}\right)$ denotes the closed convex hull of $V_{n}$. By Theorem 2.3, $W_{n}$ is a weakly compact set. Moreover from the fact that $\Gamma_{n}\left(Q_{n}\right) \subset Q_{n}$ and that $Q_{n}$ is a convex closed set we have that $W_{n} \subset Q_{n}$ and hence

$$
\Gamma_{n}\left(W_{n}\right)=\Gamma_{n}\left(\overline{c o}\left(\Gamma_{n}\left(Q_{n}\right)\right)\right) \subseteq \Gamma_{n}\left(Q_{n}\right) \subseteq{\overline{\Gamma_{n}\left(Q_{n}\right)}}^{w}=V_{n} \subset W_{n}
$$

Moreover from Proposition 4.2 and from Corollary 2.1 we obtain that the restriction of the multimap $\Gamma_{n}$ on $W_{n}$ has a weakly closed graph, hence, by Propositions 4.3 and 4.4 , it is weakly u.s.c (see [20, Theorem 1.1.5]). The conclusion then follows by Theorem 2.1. 
Remark 4.1. Suppose, for example, that there exist $\alpha \in L^{1}\left([a, b] ; \mathbb{R}_{+}\right)$and a nondecreasing function $\beta:[0,+\infty) \rightarrow[0,+\infty)$ such that $\|F(t, c)\| \leq \alpha(t) \beta(\|c\|)$ for a.e. $t \in[a, b]$ and every $c \in E$. Then condition (4.3) is equivalent to

$$
\liminf _{n \rightarrow \infty} \frac{\beta(n)}{n}=0
$$

We are able to prove the controllability result also under less restrictive growth assumptions, for instance sublinearity.

Theorem 4.2. Under assumptions $(A),(F 1),(F 2),(B),(W)$ and $\left(F 3^{\prime \prime}\right)$ there exists $\alpha \in L^{1}\left([a, b] ; \mathbb{R}_{+}\right)$such that

$$
\|F(t, c)\| \leq \alpha(t)(1+\|c\|) \text { for a.a. } t \in[a, b], \forall c \in E
$$

and

$$
M\left(1+M M_{1} M_{2} \sqrt{b-a}\right)\|\alpha\|_{1}<1
$$

controllability problem (1.1), (3.2) has a solution.

Proof. Reasoning as in Theorem 4.1 and assuming that there exist $\left\{z_{n}\right\},\left\{y_{n}\right\}$ such that $z_{n} \in Q_{n}, y_{n} \in \Gamma\left(z_{n}\right)$ and $y_{n} \notin Q_{n}, \forall n \in \mathbb{N}$, we would get

$$
\begin{aligned}
n & <\left\|y_{n}\right\|_{0} \leq C_{1}+C_{2}\left(\int_{a}^{b} \alpha(\eta)\left(1+\| z_{n}(\eta)\right) \| d \eta\right) \\
& \leq C_{1}+C_{2}(1+n)\|\alpha\|_{1}, \quad n \in \mathbb{N}
\end{aligned}
$$

giving the contradiction with (4.6).

The conclusion then follows by Theorem 2.1, like in Theorem 4.1.

Furthermore we are able to consider also superlinear growth condition, as next theorem shows.

Theorem 4.3. Assume $(A),(F 1),(F 2),(B),(W)$. If

$\left(F 3^{\prime \prime \prime}\right)$ there exist $\alpha \in L^{1}\left([a, b] ; \mathbb{R}_{+}\right)$and a nondecreasing function $\beta:[0,+\infty)$ $\rightarrow[0,+\infty)$ such that

$$
\|F(t, c)\| \leq \alpha(t) \beta(\|c\|) \text { for a.e. } t \in[a, b], \forall c \in E
$$

and $L>0$ such that

$$
\frac{L}{C_{1}+C_{2}\|\alpha\|_{1} \beta(L)}>1
$$

where $C_{1}$ and $C_{2}$ are the positive constants defined in (4.4) and (4.5), then controllability problem (1.1), (3.2) has a solution.

Proof. It is sufficient to prove that the operator $\Gamma$ maps the ball $Q_{L}$ into itself. In fact, given any $z \in Q_{L}$ and $y \in \Gamma(z)$, it holds

$$
\|y\|_{0} \leq C_{1}+C_{2}\left(\int_{a}^{b} \alpha(\eta) \beta(\|z(\eta)\|) d \eta\right) \leq C_{1}+C_{2}\|\alpha\|_{1} \beta(L)<L .
$$

The conclusion then follows by Theorem 2.1, like in Theorem 4.1. 
Remark 4.2. If, in the previous theorem, we take $\beta(s)=s^{2}, s>0$, then it is easy to prove that condition (4.7) is satisfied taking

$$
\frac{1-\sqrt{1-4 C_{1} C_{2}\|\alpha\|_{1}}}{2 C_{2} \|\left.\alpha\right|_{1}}<L<\frac{1+\sqrt{1-4 C_{1} C_{2}\|\alpha\|_{1}}}{2 C_{2} \|\left.\alpha\right|_{1}}
$$

provided $4 C_{1} C_{2}\|\alpha\|_{1}<1$.

\section{Applications}

\subsection{Nonlocal diffusion model}

In this subsection we are interested in a model of population dispersal. More precisely we study the controllability of the system governed by the following hyperbolic integro-differential inclusion, representing a model in nonlocal spatial dispersal

$$
\begin{cases}z_{t}(t, x) \in \gamma(t, x) z(t, x)+\left[f_{1}\left(t, x, \int_{\Omega} k(x, \xi) z(t, \xi) d \xi\right),\right. \\ \left.f_{2}\left(t, x, \int_{\Omega} k(x, \xi) z(t, \xi) d \xi\right)\right]+u(t, x) & x \in \Omega, 0 \leq t \leq b \\ z(0, x)=z_{0}(x) & x \in \Omega,\end{cases}
$$

where $\Omega$ is a bounded domain in $\mathbb{R}^{n}$ with a sufficiently regular boundary.

Recently, integro-differential equations have been applied to study biological invasions and disease spread. Inclusion (5.1) describes population dispersal better than ordinary differential/difference equations or reaction-diffusion equations, because it takes into account the long-distance dispersal and describes the dispersion via a dispersal kernel, which specifies the probability that an individual moves from one location to another. The function $z(t, x)$ represents the density of infective individuals at point $x$ and time $t$, while $\gamma$ is the transmission rate of infection and $k(x, y)$ is the dispersal kernel, i.e., the density function that prescribes the proportion of infective individuals leaving $y$ to $x$. The multivalued term in (5.1) represents the external influence on the process which is known up to some degree of uncertainty.

We consider controllability problem for (5.1) under the following hypotheses:

(i) For all $c \in \mathbb{R}, i=1,2, f_{i}(\cdot, \cdot, c):[0, b] \times \Omega \rightarrow \mathbb{R}$ is measurable;

(ii) For a.a. $t \in[0, b]$ and $x \in \Omega, f_{1}(t, x, \cdot): \mathbb{R} \rightarrow \mathbb{R}$ is lower semicontinuous and $f_{2}(t, x, \cdot): \mathbb{R} \rightarrow \mathbb{R}$ is upper semicontinuous;

(iii) $f_{1}(t, x, c) \leq f_{2}(t, x, c)$ in $[0, b] \times \Omega \times \mathbb{R}$;

(iv) There exist $\eta \in L^{1}([0, b] ; \mathbb{R})$ and a non decreasing function $\lambda:[0, \infty) \rightarrow$ $[0, \infty)$ such that, for a.a. $t \in[0, b]$, every $x \in \Omega, c \in \mathbb{R}$ and $i=1,2$, we have $\left|f_{i}(t, x, c)\right| \leq \eta(t) \lambda(|c|)$ with

$$
\liminf _{c \rightarrow \infty} \frac{\lambda(c)}{c}=0
$$


(v) $k: \Omega \times \Omega \rightarrow \mathbb{R}$ is measurable with $k(x, \cdot) \in L^{2}(\Omega ; \mathbb{R})$ and $\|k(x, \cdot)\|_{2} \leq 1$ for all $x \in \Omega$;

(vi) $\gamma:[0, b] \times \Omega \rightarrow \mathbb{R}$ is measurable with $\gamma(\cdot, x) \in L^{1}([0, b] ; \mathbb{R})$ and $\|\gamma(\cdot, x)\|_{1} \leq$ $\bar{\gamma}$ for every $x \in \Omega$ and some $\bar{\gamma}>0$.

Relations (5.1) can be represented in the form of the following control system in the Banach space $E=L^{2}(\Omega ; \mathbb{R})$

$$
\left\{\begin{array}{l}
y^{\prime}(t) \in A(t) y(t)+F(t, y(t))+B w(t) \\
y(0)=y_{0}
\end{array}\right.
$$

where $y:[0, b] \rightarrow E$ is defined as $y(t)=z(t, \cdot), y_{0}=z_{0}(\cdot), F:[0, b] \times E \multimap$ $E$ is $F(t, y)(x)=\left[f_{1}\left(t, x, \int_{\Omega} k(x, \xi) y(\xi) d \xi\right), f_{2}\left(t, x, \int_{\Omega} k(x, \xi) y(\xi) d \xi\right)\right], B$ is the identity operator on $E, w(t)=u(t, \cdot)$ and $\{A(t)\}_{t \in[a, b]}$ is the family of bounded linear operators in $E$ generating the evolution system

$$
T(t, s) z(x)=e^{\int_{s}^{t} \gamma(\xi, x) d \xi} z(x) .
$$

Trivially this system is never compact.

Let us show that Theorem 4.1 can be applied to the abstract formulation (5.3) of the controllability problem for system (5.1). According to Pettis measurability Theorem (see Theorem 2.4), it is possible to show that the maps $t \mapsto f_{i}\left(t, \cdot, \int_{\Omega} k(\cdot, s) y(s) d s\right), i=1,2$ are measurable selections of $F(\cdot, y)$ for every $y \in L^{2}(\Omega ; \mathbb{R})$; hence condition (F1) is satisfied.

Now we verify condition (F2). Fix $t \in[0, b]$ and consider the sequences $\left\{y_{n}\right\},\left\{\beta_{n}\right\} \subset L^{2}(\Omega ; \mathbb{R})$ satisfying $y_{n} \rightarrow y, \beta_{n} \rightarrow \beta$ in $L^{2}(\Omega ; \mathbb{R})$ and $\beta_{n} \in$ $F\left(t, y_{n}\right)$ for all $n \in \mathbb{N}$. Condition (v) implies that, for every $x \in \Omega, \int_{\Omega} k(x, \xi) y_{n}$ $(\xi) d \xi \rightarrow \int_{\Omega} k(x, \xi) y(\xi) d \xi$ and

$$
\left|\int_{\Omega} k(x, \xi) y_{n}(\xi) d \xi\right| \leq\|k(x, \cdot)\|_{2}\left\|y_{n}\right\|_{2} \leq\left\|y_{n}\right\|_{2} .
$$

Since $\beta_{n} \rightarrow \beta$, applying Mazur's convexity lemma, we have the existence of a sequence

$$
\tilde{\beta}_{n}=\sum_{i=0}^{k_{n}} \delta_{n, i} \beta_{n+i} \quad \delta_{n, i} \geq 0, \sum_{i=0}^{k_{n}} \delta_{n, i}=1
$$

such that $\tilde{\beta}_{n} \rightarrow \beta$ in $L^{2}(\Omega ; \mathbb{R})$ and up to a subsequence denoted as the sequence $\tilde{\beta}_{n}(x) \rightarrow \beta(x)$ for a.a. $x \in \Omega$. By definition we have, for a.a. $x \in \Omega$,

$$
\begin{aligned}
& \sum_{i=0}^{k_{n}} \delta_{n, i} f_{1}\left(t, x, \int_{\Omega} k(x, \xi) y_{n+i}(\xi) d \xi\right) \\
& \quad \leq \tilde{\beta}_{n}(x) \leq \sum_{i=0}^{k_{n}} \delta_{n, i} f_{2}\left(t, x, \int_{\Omega} k(x, \xi) y_{n+i}(\xi) d \xi\right)
\end{aligned}
$$

and passing to the limit as $n \rightarrow \infty$, according to (ii), we obtain that $\beta \in F(t, y)$. We have showed that $F(t, \cdot)$ has weakly closed graph. Moreover, according to 
(5.5) and (iv), for every $i=1,2, x \in \Omega$ and $y \in E$,

$$
\left|f_{i}\left(t, x, \int_{\Omega} k(x, \xi) y(\xi) d \xi\right)\right| \leq \eta(t) \lambda\left(\|y\|_{2}\right)
$$

implying

$$
\|F(t, y)\|_{2}^{2} \leq \eta(t)^{2} \lambda\left(\|y\|_{2}\right)^{2}|\Omega|
$$

and so the growth condition $\left(\mathrm{F} 3^{\prime}\right)$ holds with $\omega_{n}(t)=\eta(t) \lambda(n) \sqrt{|\Omega|}$. Hence, (5.2) yields

$$
\liminf _{n \rightarrow \infty} \frac{1}{n} \int_{0}^{b} \omega_{n}(t) d t=\liminf _{n \rightarrow \infty} \frac{\lambda(n)}{n}\|\eta\|_{1} \sqrt{|\Omega|}=0
$$

and hence (4.3) is fulfilled.

Trivially, (B) is verified. Let us now show (W). Given $z \in L^{2}(\Omega ; \mathbb{R})$, let us consider the measurable function $u_{z}:[0, b] \rightarrow L^{2}(\Omega ; \mathbb{R})$ defined as

$$
u_{z}(t)(x)=\frac{1}{b} e^{-\int_{t}^{b} \gamma(\xi, x) d \xi} z(x) .
$$

Since, for every $t \in[0, b], x \in \Omega$ and $z \in L^{2}(\Omega ; \mathbb{R})$, (vi) implies

$$
\left|u_{z}(t)(x)\right| \leq \frac{1}{b} e^{b \bar{\gamma}}|z(x)|,
$$

it follows that $u_{z} \in L^{2}([0, b] ; E)$. Moreover, for every $s \in[0, b]$

$$
T(b, s) B u_{z}(s)=\frac{1}{b} T(b, s) e^{-\int_{s}^{b} \gamma(\xi, \cdot) d \xi} z=\frac{1}{b} e^{\int_{s}^{b} \gamma(\xi, \cdot) d \xi} e^{-\int_{s}^{b} \gamma(\xi, \cdot) d \xi} z=\frac{1}{b} z,
$$

therefore

$$
\int_{0}^{b} T(b, s) B u_{z}(s) d s=\frac{1}{b} \int_{0}^{b} z d s=z,
$$

i.e. for every $z \in L^{2}(\Omega ; \mathbb{R})$ there exists $u_{z} \in L^{2}([0, b] ; E)$ such that $W u_{z}=z$. We obtain that all the assumptions of Theorem 4.1 hold, implying that the controllability problem for system (5.1) has a solution $z \in C\left([0, b] ; L^{2}(\Omega ; \mathbb{R})\right)$.

\subsection{Telegraph equation}

We study the controllability problem for the system governed by the second order one-dimensional telegraph equation with constant coefficients, modeled by the following hyperbolic integro-differential equation

$$
\left\{\begin{array}{l}
z_{t t}=z_{x x}+f\left(t, x, \int_{0}^{\ell} k(x, \xi) z(t, \xi) d \xi\right)+L u(t, x), \quad 0 \leq x \leq \ell, \quad 0 \leq t \leq b \\
z(t, 0)=0 \quad 0 \leq t \leq b, \\
z(0, x)=z_{0}(x), z_{t}(0, x)=z_{1}(x) \quad 0 \leq x \leq \ell .
\end{array}\right.
$$

Both the electric voltage and the current in a double conductor satisfy equation (5.6). The interacting quantity $f$ contains an integral term introduced to 
take into account the effects of finite velocity to standard heat or mass transport equation.

We assume the following hypotheses:

(i) $f(\cdot, \cdot, c):[0, b] \times[0, \ell] \rightarrow \mathbb{R}$ is measurable, for all $c \in \mathbb{R}$;

(ii) $f(t, x, \cdot): \mathbb{R} \rightarrow \mathbb{R}$ is continuous, for a.a. $(t, x) \in[0, b] \times[0, \ell]$;

(iii) There exist $\eta \in L^{1}([0, b] ; \mathbb{R})$ and $\lambda:[0, \infty) \rightarrow[0, \infty)$ increasing such that, for a.a. $t \in[0, b]$ and every $x \in[0, \ell], c \in \mathbb{R},|f(t, x, c)| \leq \eta(t) \lambda(|c|)$ and

$$
\liminf _{c \rightarrow \infty} \frac{\lambda(c)}{c}=0
$$

(iv) $k:[0, \ell] \times[0, \ell] \rightarrow \mathbb{R}$ is measurable with $k(x, \cdot) \in L^{2}([0, l] ; \mathbb{R})$ and $\|k(x, \cdot)\|_{2}$ $\leq 1$ for all $x \in[0, \ell]$

(v) The control $w(t)=u(t, \cdot)$ belongs to $L^{2}\left([0, b] ; L^{2}([0, \ell] ; \mathbb{R})\right)$;

(vi) The operator $L: \mathbb{R} \rightarrow \mathbb{R}$ is such that $(B w)(x)=L w(x)$ is a bounded surjective operator in $L^{2}([0, \ell] ; \mathbb{R})$.

Again, we rewrite Eq. (5.6) as the control system (5.3) in the Banach space $E=L^{2}([0, \ell] ; \mathbb{R})$. In fact, using the same notation as before, problem (5.6) can be rewritten as a second order equation of the following form

$$
\left\{\begin{array}{l}
y^{\prime \prime}(t)=A y(t)+F(t, y(t))+B w(t), t \in[0, b] \\
y(0)=y_{0} ; y^{\prime}(0)=y_{1}
\end{array}\right.
$$

where $y_{1}=u_{1}(\cdot)$ and $A: D(A)=\left\{y \in W^{2,2}([0, \ell] ; \mathbb{R}): y(0)=y(\ell)=0\right\} \rightarrow$ $L^{2}([0, \ell] ; \mathbb{R})$ is the Laplace operator $A y=y^{\prime \prime}$.

Observe that $-A$ is a self-adjoint and positive definite operator on $L^{2}([0, \ell] ; \mathbb{R})$ with a compact inverse, hence there exists a unique positive definite square root $(-A)^{1 / 2}$ with domain $D\left((-A)^{1 / 2}\right)=\left\{y \in W^{1,2}([0, \ell] ; \mathbb{R})\right.$ : $y(0)=y(\ell)=0\}$ (see, e.g. [17]). Therefore, denoting by $\mathcal{E}$ the Banach space $W_{0}^{1,2}([0, \ell] ; \mathbb{R}) \times L^{2}([0, \ell] ; \mathbb{R})$ and $\mathcal{A}: \mathcal{E} \rightarrow \mathcal{E}$ the linear operator defined by

$$
\mathcal{A}=\left(\begin{array}{ll}
0 & I \\
A & 0
\end{array}\right),
$$

we can treat (5.7) as a first order semilinear differential equation in $\mathcal{E}$

$$
\left\{\begin{array}{l}
r^{\prime}(t)=\mathcal{A} r(t)+\mathcal{F}(t, r(t))+\mathcal{B} p(t), t \in[0, b] \\
r(0)=\left(\begin{array}{l}
y_{0} \\
y_{1}
\end{array}\right)
\end{array}\right.
$$

where $\mathcal{F}:[0, b] \times \mathcal{E} \rightarrow \mathcal{E}$ is defined as $\mathcal{F}\left(t,\left(r_{1}, r_{2}\right)\right)=\left(0, F\left(t, r_{1}\right)\right)$ and $\mathcal{B}: \mathcal{E} \rightarrow \mathcal{E}$ can be expressed as $\mathcal{B}\left(p_{1}, p_{2}\right)=\left(0, B p_{1}\right)$.

We now prove that Theorem 4.1 can be applied to controllability problem for (5.8), obtaining as the result a solution of the corresponding problem for (5.6). Notice first of all that Pettis measurability theorem (see [29, p. 278]), the separability of $L^{2}([0, \ell] ; \mathbb{R})$ and conditions (i) and (ii) imply that $F$ is measurable (see [20, Corollary 1.3.1]) and, being single-valued, it satisfies (F1). 
Fix now $t \in[0, b]$ satisfying (ii) and take $z_{n} \rightarrow z$ in $L^{2}([0, \ell] ; \mathbb{R})$. Then, for every $x \in[0, \ell]$, it holds $\int_{0}^{\ell} k(x, \xi) z_{n}(\xi) d \xi \rightarrow \int_{0}^{\ell} k(x, \xi) z(\xi) d \xi$, thus condition (ii) implies that $f\left(t, x, \int_{0}^{\ell} k(x, \xi) z_{n}(\xi) d \xi\right) \rightarrow f\left(t, x, \int_{0}^{\ell} k(x, \xi) z(\xi) d \xi\right)$ for a.a. $x \in[0, \ell]$. Observe that for every $x \in[0, \ell]$ and $z \in L^{2}([0, \ell] ; \mathbb{R})$, according to (iv), the following estimate holds

$$
\left|\int_{0}^{\ell} k(x, \xi) z(\xi) d \xi\right| \leq\|k(x, \cdot)\|_{2}\|z\|_{2} \leq\|z\|_{2} .
$$

Therefore, since the weak convergence of $\left\{z_{n}\right\}$ yields its boundedness, according to (iii), there exists a constant $M>0$ such that, for every $n \in \mathbb{N}$,

$$
\left|f\left(t, \cdot, \int_{0}^{\ell} k(\cdot, \xi) z_{n}(\xi) d \xi\right)\right| \leq \eta(t) \lambda\left(\left\|z_{n}\right\|_{2}\right) \leq \eta(t) \lambda(M)
$$

and Lebesgue Convergence Theorem yields that $f\left(t, \cdot, \int_{0}^{\ell} k(\cdot, \xi) z_{n}(\xi) d \xi\right) \rightarrow$ $f\left(t, \cdot, \int_{0}^{\ell} k(\cdot, \xi) z(\xi) d \xi\right)$ in $L^{2}([0, \ell] ; \mathbb{R})$ verifying condition $(\mathrm{F} 2)$.

Finally, according to (iii) and (5.9), we have, for a.a. $t \in[0, b]$ and every $z \in L^{2}([0, \ell] ; \mathbb{R})$,

$$
\|F(t, z)\|_{2}^{2}=\int_{0}^{\ell}\left[f\left(t, x, \int_{0}^{\ell} k(x, \xi) z(\xi) d \xi\right)\right]^{2} d x \leq \eta(t)^{2} \lambda\left(\|z\|_{2}\right)^{2} \ell .
$$

So, the growth condition $\left(\mathrm{F} 3^{\prime}\right)$ and (4.3) hold with $\omega_{n}(t)=\eta(t) \lambda(n) \sqrt{\ell}$, indeed condition (iii) implies

$$
\liminf _{n \rightarrow \infty} \frac{1}{n} \int_{0}^{b} \omega_{n}(t) d t=\liminf _{n \rightarrow \infty} \frac{\lambda(n)}{n}\|\eta\|_{1} \sqrt{\ell}=0 .
$$

Moreover conditions (v) and (vi) yield (B) and (W) (see [17, Example VI.8.10]) and hence we obtain a solution $z \in C\left([0, b] ; L^{2}([0, \ell] ; \mathbb{R})\right)$ of the controllability problem for (5.6).

\section{Acknowledgements}

The work of V. Obukhovskii was supported by the Russian FBR Grant 14-0100468. The work of I. Benedetti and V. Taddei was supported by the project PRIN-MIUR 2009 Ordinary Differential Equations and Applications and by the National Research Project GNAMPA 2013 Metodi Topologici per Problemi Differenziali Nonlineari e Applicazioni.

\section{References}

[1] Arutyunov, A.V., Aseev, S.M., Blagodatskih, V.I.: Necessary conditions of the first order in the problem of the optimal control of a differential inclusion with phase constraints. Russian Acad. Sci. Sb. Math. 79(1), 117-139 (1994) 
[2] Arutyunov, A.V., Blagodatskih, V.I.: The maximum principle for differential inclusions with phase constraints. Proc. Steklov Inst. Math. 2(200), 3-25 (1993)

[3] Balachandran, K., Dauer, J.P.: Controllability of nonlinear systems in Banach spaces: a survey. J. Optim. Theory Appl. 115(1), 7-28 (2002)

[4] Benchohra, M., Gatsori, E.P., Ntouyas, S.K.: Controllability results for semilinear evolution inclusions with nonlocal conditions. J. Optim. Theory Appl. 118(3), 493-513 (2003)

[5] Benedetti, I., Malaguti, L., Taddei, V.: Two-point b.v.p. for multivalued equations with weakly regular r.h.s.. Nonlinear Anal. 74, 3657-3670 (2011)

[6] Benedetti, I., Malaguti, L., Taddei, V.: Semilinear evolution equations in abstract spaces and applications. Rend. Ist. Matem. Un. Trieste (Special issue dedicated to the 60th birthday of Prof. F. Zanolin) 44, 371-388 (2012)

[7] Benedetti, I., Malaguti, L., Taddei, V.: Nonlocal semilinear evolution equations without strong compactness: theory and applications. Bound. Value Probl. (Special Issue Dedicated to Jean Mawhin's Achievements in Nonlinear Analysis) 60, 1-18 (2013)

[8] Benedetti, I., Obukhovskii, V.V., Zecca, P.: Controllability for impulsive semilinear differential inclusions in a Banach space with a non-compact semigroup. Disc. Math. Diff. Incl. Control Optim. 31, 39-69 (2011)

[9] Bochner, S., Taylor, A.E.: Linear functionals on certain spaces of abstractlyvalued functions. Ann. Math. 39(4), 913-944 (1938)

[10] Brezis, H.: Analyse fonctionelle, théorie et applications. Masson Editeur, Paris (1983)

[11] Burton, T.A.: Volterra integral and differential equations. In: Mathematics in science and engineering 202. Elsevier, Amsterdam (2005)

[12] Cardinali, T., Rubbioni, P., Portigiani, F.: Nonlocal Cauchy problems and their controllability for semilinear differential inclusions with lower Scorza-Dragoni nonlinearities. Czech. Math. J. 61(136)(1), 225-245 (2011)

[13] Corduneanu, C.: Integral equations and applications. Cambridge University Press, Cambridge (1991)

[14] Dauer, J.P., Mahmudov, N.I.: Controllability of some nonlinear systems in Hilbert spaces. J. Optim. Theory Appl. 123(2), 319-329 (2004)

[15] Dunford, N., Schwartz, J.T.: Linear operators. Wiley, New York (1988)

[16] Ekeland, I., Teman, R.: Convex anaysis and variational problems. North Holland, Amstrdam (1976)

[17] Engel, K.J., Nagel, R.: One-parameter semigroups for linear evolution equations. In: Graduate texts in mathematics 194. Springer, New York (2000)

[18] Glicksberg, L.I.: A further generalization of the Kakutani fixed theorem with application to Nash equilibrium points. Proc. Amer. Math. Soc. 3, 170-174 (1952) 
[19] Gorniewicz, L., Ntouyas, S.K., O'Regan, D.: Controllability results for first and second order evolution inclusions with non local conditions. Ann. Pol. Math. 89(1), 65-101 (2006)

[20] Kamenskii, M., Obukhovskii, V., Zecca, P.: Condensing multivalued maps and semilinear differential inclusions in banach spaces. In: de Gruyter Series in Nonlinear Analysis and Applications, 7. Walter de Gruyter, Berlin (2001)

[21] Kantorovich, L.V., Akilov, G.P.: Functional analysis. Pergamon Press, Oxford (1982)

[22] Kisielewicz, M.: Differential inclusions and optimal control. In: Mathematics and its Applications (East European Series), 44. Kluwer, Dordrecht (1991)

[23] Krein, S.G.: Linear Differential Equations in Banach Spaces. American Mathematical Social, Providence (1971)

[24] Ky, F.: Fixed point and minimax theorems in locally convex topological linear spaces. Proc. Natl. Acad. Sci. USA 38, 121-126 (1952)

[25] Mahmudov, N.I.: Exact null controllability of semilinear evolution systems. J. Glob. Optim. 56, 317-326 (2013)

[26] Metaxas, A.C., Meredith, R.J.: Industrial microwave heating. Peter Peregrinus, London (1993)

[27] Obukhovskii, V., Zecca, P.: Controllability for systems governed by semilinear differential inclusions in a Banach space with a non-compact semigroup. Nonlinear Anal. 70(9), 3424-3436 (2009)

[28] Pazy, A.: Semigroups of linear operators and applications to partial differential equations. Springer, Berlin (1983)

[29] Pettis, B.J.: On the integration in vector spaces. Trans. Amer. Math. Soc. 44(2), 277-304 (1938)

[30] Roussy, G., Pearcy, J.A.: Foundations and industrial applications of microwaves and radio frequency fields. Wiley, New York (1995)

[31] Schwartz, L.: Cours d'Analyse I. 2nd edn. Hermann, Paris (1981)

[32] Triggiani, R.: A note on the lack of exact controllability for mild solutions in Banach spaces. SIAM J. Control Optim. 15(3), 407-411 (1977)

[33] Triggiani, R.: Addendum a note on the lack of exact controllability for mild solutions in Banach spaces. SIAM J. Control Optim. 18(1), 98-99 (1980)

Irene Benedetti

Dipartimento di Matematica e Informatica

Università degli Studi di Perugia

06123 Perugia

Italy

e-mail: irene.benedetti@dmi.unipg.it 
Valeri Obukhovskii

Faculty of Physics and Mathematics

Voronezh State Pedagogical University

394043 Voronezh

Russia

e-mail: valerio-ob2000@mail.ru

Valentina Taddei

Dip. di Scienze Fisiche, Informatiche e Matematiche Università di Modena e Reggio Emilia

41125 Modena

Italy

e-mail: valentina.taddei@unimore.it

Received: 4 December 2013.

Accepted: 5 March 2014. 